\title{
Evaluation of Multichannel Synchronous Conducted TDEMI Measurements for High Voltage Power Electronics
}

\author{
Tom Hartman*, Niek Moonen*, Frank Leferink* ${ }^{* \dagger}$ \\ ${ }^{*}$ University of Twente, Enschede, Netherlands, t.h.f.hartman@ @student.utwente.nl \\ ${ }^{\dagger}$ Thales Nederland B.V., Hengelo, Netherlands
}

\begin{abstract}
Safely measuring high power conducted electromagnetic interference (EMI) is an issue to be addressed, where a possible measurement strategy is being discussed in this paper which uses the benefits of multi-channel synchronous timedomain electromagnetic interference (TDEMI) measurements. Only the differential mode (DM) voltage has been evaluated in this paper, however the setup is not limited in this respect. Common mode (CM) voltage can also be synchronously analyzed with this setup. Nevertheless, with respect to the to be measured amplitudes, DM voltages in this particular system offer a larger challenge and are addressed. The setup was developed with respect to Low-Frequency conducted EMI measurements in high power, fast switching systems using a low-cost solution.
\end{abstract}

\section{INTRODUCTION}

As a result of the emerging new technologies and the rapid development of new electronic products, the ability to achieve electromagnetic compatibility and to improve it becomes a major challenge in the development of newer electronic products. Equipment to quickly and fully characterize a system's electromagnetic compatibility will result in a decrease of the costs of the system and it will also improve the quality in circuit and system development. Traditionally radio noise and electromagnetic interference were measured and characterized using superheterodyne radio receivers, which require measurement bandwidths, step sizes and dwell time. As has been shown in [1], this can result in extreme measurement times. The proposed solution is time-domain measurements in combination with digital signal processing (DSP).

Several time-domain electromagnetic interference (TDEMI) measurements have been studied extensively in for instance [2]-[4], with an increasing interest in the (fast) transient analysis [5], [6]. With [2] proposing to decompose the electromagnetic interference (EMI) in differential mode (DM) and common mode (CM), [5] decomposing the EMI transient phenomena in time domain through DSP, and [3], [6] to use short term fast Fourier transform (STFFT) techniques to present time-frequency plots (i.e. spectrograms). The TDEMI approach, as an alternative for EMI receiver, benefits [1], [4] and challenges [7], [8] have been addressed.

In this paper, the TDEMI approach is used for determining conducted interference originating from a Galium-Nitride $(\mathrm{GaN})$ based DC/AC converter. As today's commercial of the shelf (COTS) power electronics have switching frequencies within the range of $2 \mathrm{kHz}-150 \mathrm{kHz}$, for which civil emission standards are lacking [9], the low frequency (DC-30 MHz) conducted EMI frequency band is of great interest. This paper will focus on the evaluation of multichannel synchronous measurements as stated in [4] and the possible challenges it brings. Fig. 1 shows a quick overview of the measured parameters and used method.

An 8-channel scope is used in conjunction with the 'mains monitor box' as described in [9] and will be elaborated on in Section II. The goal is to compare different methods of measuring and calculating, via DSP, the DM voltage. Three separate methods are used to investigate the DM EMI:

- Symmetric Voltage Measurement $\left(V_{d m}\right)$

- $\Delta$ Non-Symmetric Voltage Measurement $\left(V_{L}-V_{N}\right)$

- $\Delta$ Non-Symmetric Current Measurement $\left(I_{L}-I_{N}\right)$

Voltage and current measurements have an overlapping bandwidth from approximately $2 \mathrm{kHz}$ to $100 \mathrm{kHz}$.

In the following section the measurement setup is briefly discussed, which is then followed by a discussion on the beneficial effect of multi-channel measurements. The results presented in section IV are qualitatively reviewed, as this is a proof-of-concept type of setup.

\section{Measurement Setup}

Safely measuring DM EMI in a relatively high voltage (i.e. above $100 \mathrm{~V}$ ) setup is done as depicted in Fig. 2a. The inside of a conducted measurement device, as seen in Fig. 2a, can be seen in Fig. 2b and Fig. 2c. As the emphasis of this paper lies in synchronous time domain measurements, the functional behavior of the AC/DC converter will only be briefly described here.

\section{A. GaN Half-bridge}

In Fig. 2a it can be seen that the 'DC' source is a galvanic isolated grid that has been rectified. By using sinusoidal pulse width modulation (sPWM) driver logic, the switches are operated in such a way, that the switching node is either connected to the $+165 \mathrm{~V}$ or $-165 \mathrm{~V}$. This results in a sPWM voltage waveform that contains two main frequency components, $f_{c}$ and $f_{m}$, which are the switching frequency and $\mathrm{AC}$ output frequency respectively. [10], [11] describe the full background for studying a DC/AC converter with extreme flexibility in choosing these frequencies. However, in the evaluated setup $f_{c}=25 \mathrm{kHz}$ and $f_{m}=50 \mathrm{~Hz}$, which implies that, when 


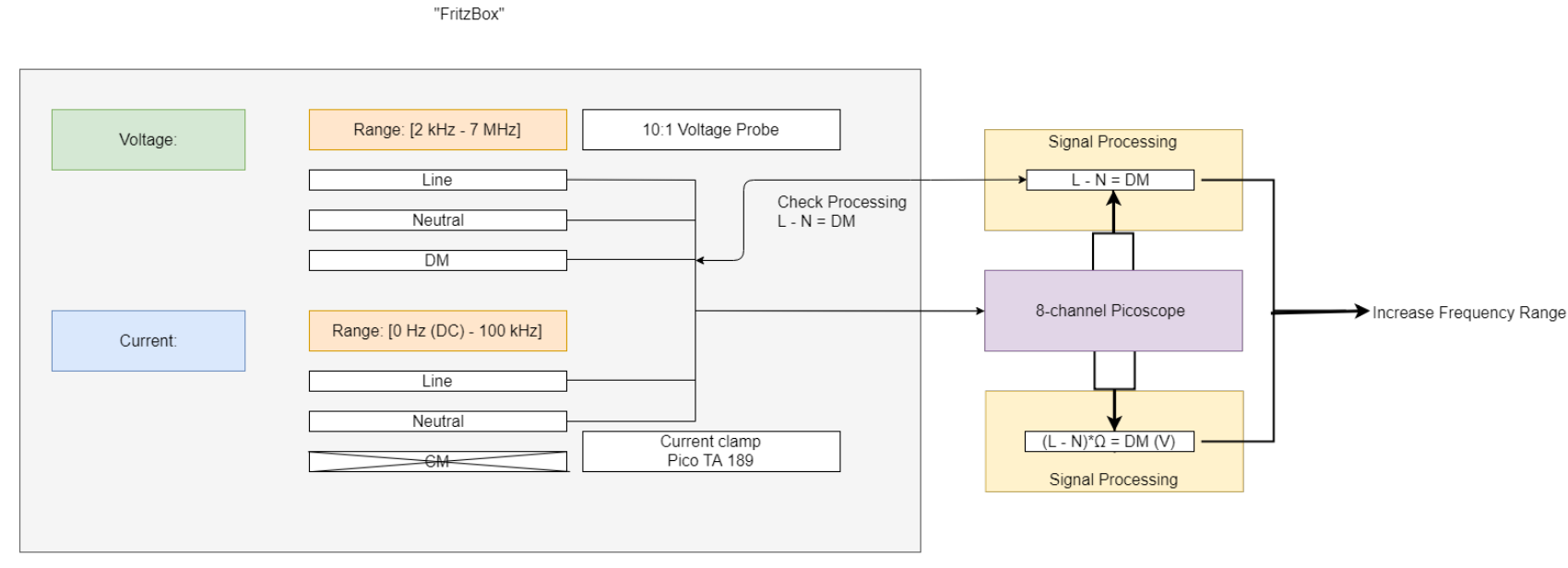

Fig. 1: General Overview

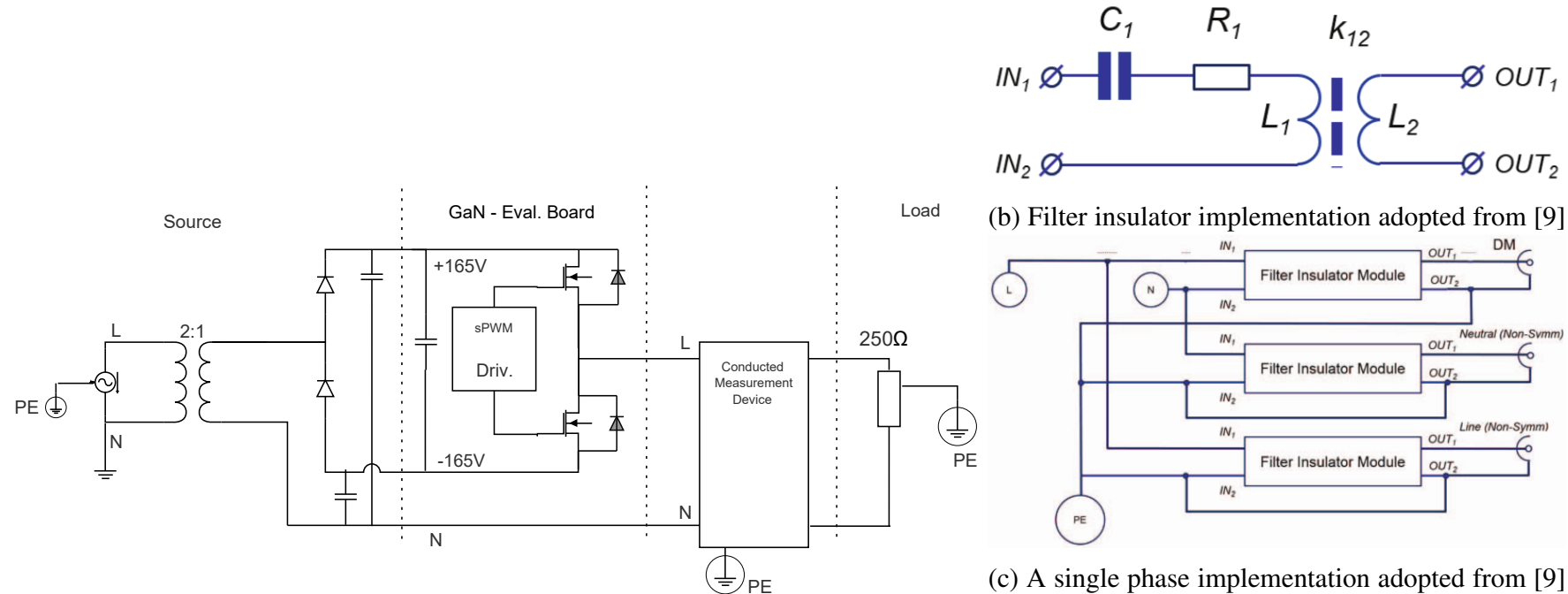

(a) setup

Fig. 2: Schematic representation of the conducted emission test setup

the output waveform is low pass filtered, the resulting $\mathrm{AC}$ signal consists only of a $50 \mathrm{~Hz}$ component with an approximate $V_{r m s}=116 \mathrm{~V}$.

\section{B. Five channels}

As the setup is evaluated for the use in operational high power switching electronics, three methods for determining the DM EMI were applied, which require the synchronous measurement of five separate parameters:

- $V_{d m}$ : Differential mode Voltage

- $V_{L}$ : Line Voltage

- $V_{N}$ : Neutral Voltage

- $I_{L}$ : Line Current

- $I_{N}$ : Neutral Current

The used low-cost PicoScope functioning as an oscilloscope, has a maximum input voltage range of $\pm 50 \mathrm{~V}$ and the possibil-
TABLE I: Measurement Equipment

\begin{tabular}{|c|c|c|}
\hline & Pico TA 189 & "Meas. Box" \\
\hline Quantity: & Current & Voltage \\
Measured Modes: & Line and Neutral & Line, Neutral and DM \\
Frequency Range: & DC - $100 \mathrm{kHz}$ & $2 \mathrm{kHz}-7 \mathrm{MHz}$ \\
Ratio's & $1: 10$ & $1: 50$ \\
\hline
\end{tabular}

ity of measuring 8 channels at the same time. Table I summarizes the measurement equipment specifications. By combining the results from the current and voltage measurements, one is able to determine the conducted EMI from DC to $7 \mathrm{MHz}$ without endangerment of the used scope. In the following section possible beneficial effects of DSP are discussed, while in section IV the acquired DM voltages are verified with an analog separated DM voltage. 


\section{BENEFICIAL EFFECTS OF MULTI-CHANNEL SyNCHRONOUS CONDUCTED TDEMI MEASUREMENTS}

The previous section has described a type of switch mode power supply (SMPS) that is high power and has an operating frequency within the problematic low frequency conducted EMI band. This section addresses possible benefits from using a multi-channel measurement setup.

\section{A. Mode separation}

Multi-channel measurements have shown the possibility to separate CM and DM (noise) signals through DSP, by measuring the line and neutral voltages in the time domain or the line and the neutral currents. In the measurement setup it was mentioned that only the DM voltage is studied.

As shown in [3] the symmetric DM Voltage can be calculated via the line and neutral voltage as follows:

$$
V_{d m}=V_{L}-V_{N}
$$

and indirectly via the line and neutral current as:

$$
V_{d m}=\left(I_{L}-I_{N}\right) \cdot Z
$$

Where in this case $Z=250 \Omega$ and is assumed to be constant over the entire frequency range.

\section{B. Extended Frequency Range}

By using the measurement box from [9], which is depicted in Fig. 2, the voltage measurement bandwidth ranges from $2 \mathrm{kHz}$ until $7 \mathrm{MHz}$. This together with having a current meter going from DC to $100 \mathrm{kHz}$ gives rise to the possibility to increase the total (one-shot measured) frequency range with respect to a single channel measurement, while maintaining a low noise floor. In [7] it is already discussed that there are challenges when using a single A/D converter (i.e. channel) with respect to the required dynamic range. As a verification, all three methods are displayed in Fig. 7. The frequency range displayed is the overlapping range of $2 \mathrm{kHz}$ to $100 \mathrm{kHz}$.

As the results are from a 0.2 seconds measurement with a sampling rate of $40 \mathrm{MHz}$, the possibilities for applying DSP are endless. In the following section the results will be presented without applying any DSP, apart from the one mentioned in this section. The results are presented following the overview given in Fig. 1.

\section{Results}

At first the results are shown over the entire frequency range, 0 - $20 \mathrm{MHz}$. The different measurement techniques are plotted together on a logarithmic scale which can be seen in Fig. 3. Note that the signals are even plotted for frequencies outside the frequency ranges mentioned in Table I.

The next first logical step is then to only plot the respective frequency ranges of the different parts of the measurement set-up, which can be seen in Fig. 4

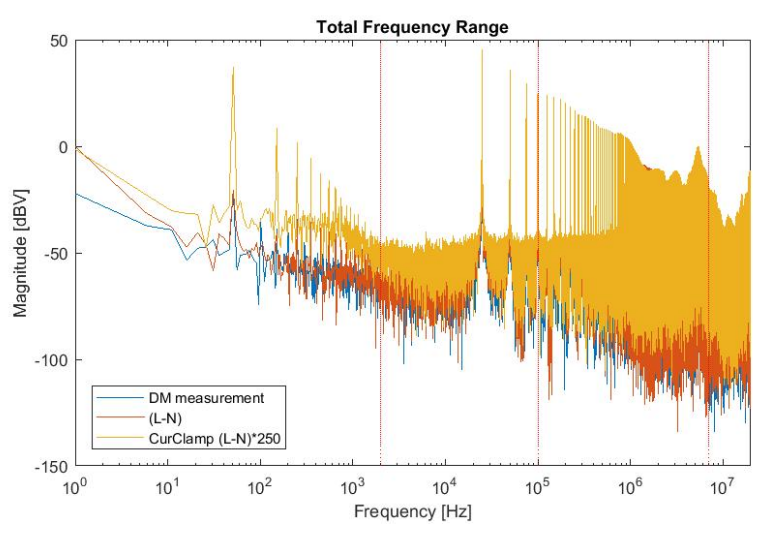

Fig. 3: Full Frequency Range

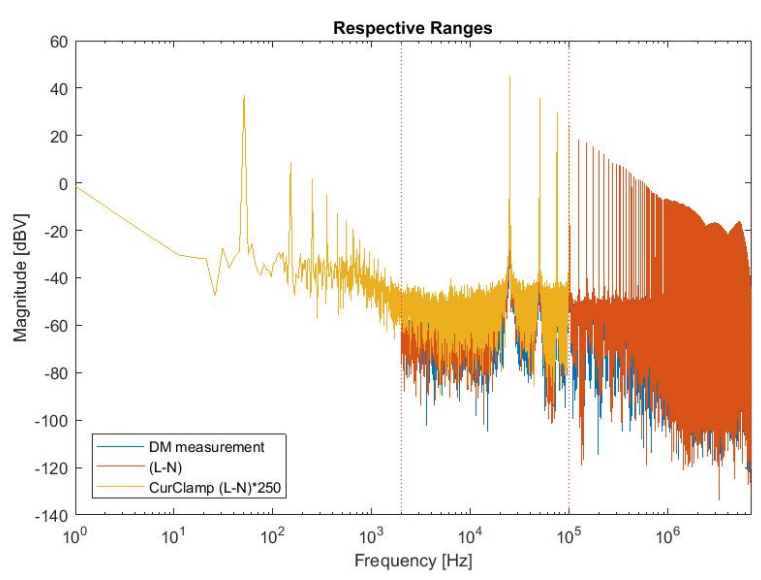

Fig. 4: Overlapping Respective Frequency Ranges

\section{A. Separation results}

Next the DM voltage measured directly and via DSP, by subtracting the neutral voltage from the line voltage, are compared. Such a comparison for their respective ranges is plotted in Fig. 5. To make a quantitative comparison the average difference in $\mathrm{dB}$ is calculated and found to be 0.3168 $\mathrm{dB}$. Note that in the case of the analog separation one measures a single voltage, while in the digital separation, two signals are measured, which has an influence on the difference in noise levels. The assumption of the 1:50 ratio in the measurement setup, mentioned in Table I and elaborated on in [9] is related to component values used, which are assumed to be $2.5 \mathrm{k} \Omega$ and $50 \Omega$. However, as with any mass produced component, they are subjected to production errors. In case of the digital separation the ratio should be equal for line and neutral voltages. If not, a larger error can be introduced here than in the case of the analog separation. The average deviation of $0.3168 \mathrm{~dB}$ might have originated from this introduced error.

\section{B. Comparison of methods}

It is easily seen from the values in Table I that there is an overlapping range for the different measurement techniques between $2 \mathrm{kHz}$ and $100 \mathrm{kHz}$. As a validation of extending 


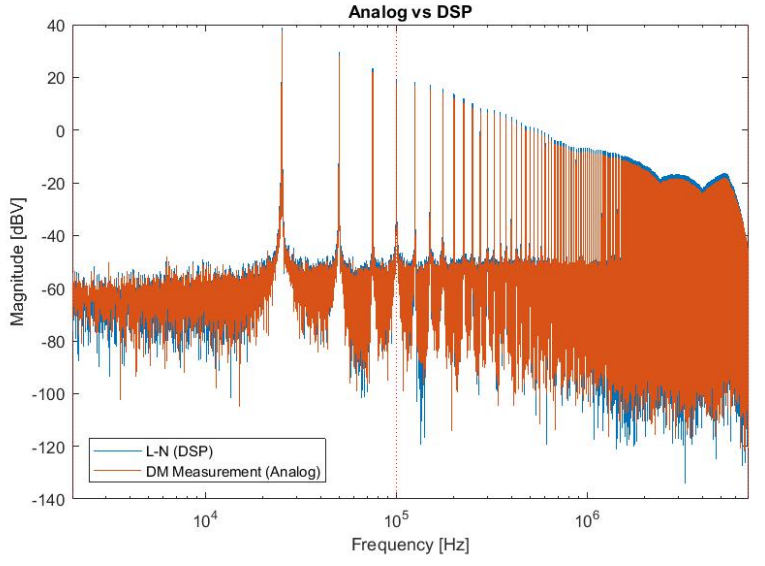

Fig. 5: Analog vs DSP

the frequency range by combining the separate measurement techniques, the found values within this range should be conform. The overlapping frequency range is plotted in Fig. 7. It can be quickly seen that the peaks are similar while using different measurement techniques. When looking at the average deviation between the signals it is found however that the calculated DM voltage via the current clamp deviates around $8 \mathrm{~dB}$. This deviation is due to the higher noise floor which is apparent in the picture. However inspection of the first peak values (at $25 \mathrm{kHz}$ ) for $I_{L-N}, V_{D M}$ and $V_{L-N}$ give $39 \mathrm{~dB}, 37 \mathrm{~dB}$ and $32 \mathrm{~dB}$ respectively. As explained earlier, the large deviation of $V_{L-N}$ might have originated from deviating component values. Comparing $I_{L-N}$ and $V_{D M}$, shows a deviation of $2 \mathrm{~dB}$, which is relatively large. In case of the current measurement, a load of $250 \Omega$ was assumed to be broadband.

Based on the above stated deviations, the voltage port transfer functions were measured to determine if the ratio is indeed 1:50. As is seen in Fig.6, there is a large deviation in the neutral port. After taking this into account, the peak values for $V_{D M}$ and $V_{L-N}$ are deviating by only $0.16 \mathrm{~dB}$.

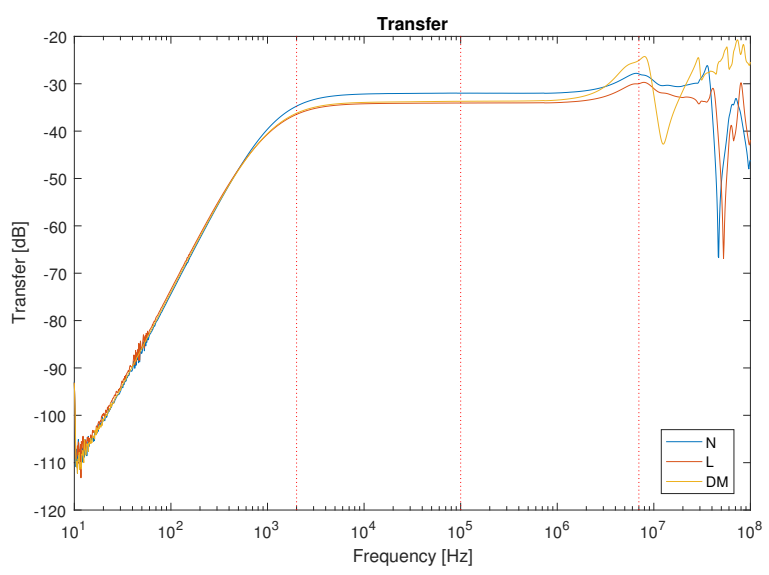

Fig. 6: Measured transfer functions of DM-, line and neutral voltage ports

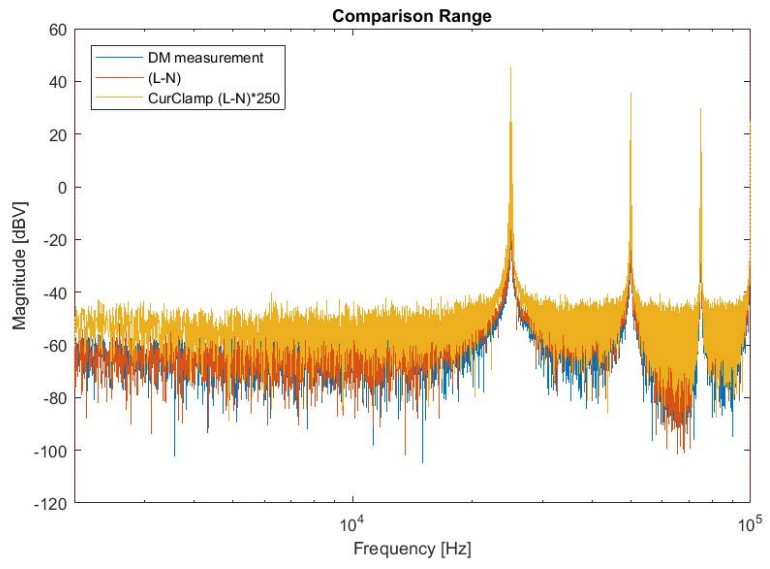

Fig. 7: Frequency Range Comparison

\section{Extended Frequency Range}

As the $V_{D M}$ and $V_{L-N}$ are deviating only slightly, the following graph, Fig. 8 is plotted using the voltage measurement results in the overlapping frequency range. This new extended frequency range is then plotted and can be seen in Fig. 9. Where the rough transition at the $2 \mathrm{kHz}$ mark is due to the previously mentioned difference in noise levels and the falsely assumed load value.

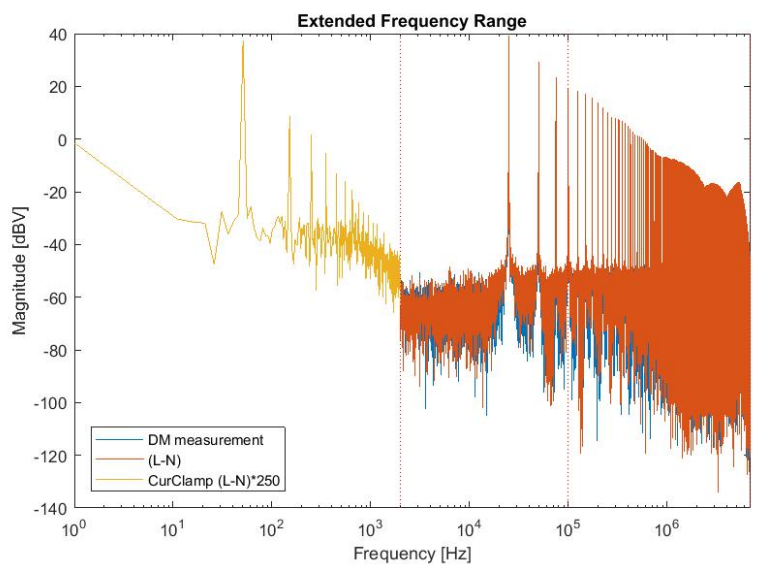

Fig. 8: Extended Frequency Range

\section{CONCLUSION}

With the aim to benefit from synchronous time domain measurements, an 8 channel measurement setup has been qualitatively evaluated. Possibilities for safely measuring high voltage applications have been discussed, with the enablement of noise mode separation. Mode separation through analog circuits as well as DSP have been discussed and found to have a negligible deviation. It has also been shown that by combining low-frequency current measurements with highfrequency voltage measurements, one can extend the frequency range of a single measurement, without compromising the signal to noise ratio due to the limited dynamic range of 


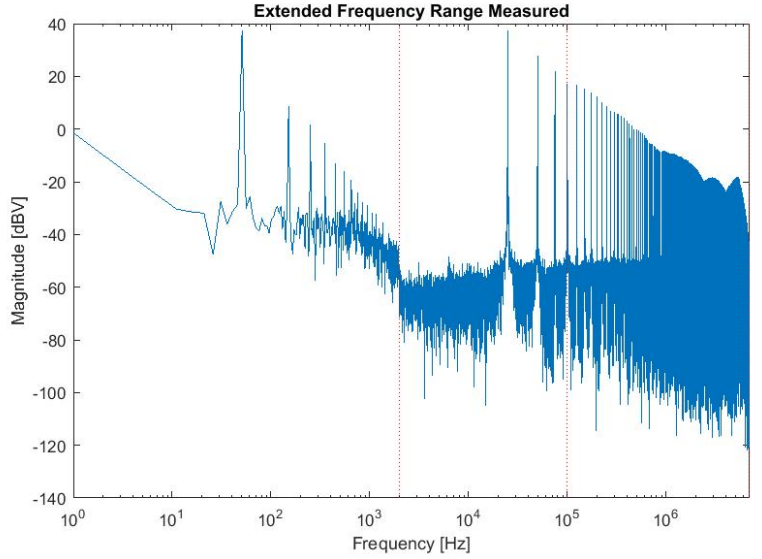

Fig. 9: 1 line Extended Frequency Range Measured DM voltage

an ADC. However in the measurement setup presented in this paper, the lack of a frequency dependent impedance measurement of the load resulted into a large deviation when comparing the overlapping frequency range. Nonetheless, it has been shown that it is possible to benefit from multi-channel synchronous conducted TDEMI measurements applied in high power electronic systems.

\section{REFERENCES}

[1] I. Setiawan, N. Moonen, F. Buesink, and F. Leferink, "Efficient Magnetic Field Measurements," 2017.

[2] Y.-s. Lee, "Time Domain Measurement System for Conducted EMI and CM/DM Noise Signal Separation," 2005 International Conference on Power Electronics and Drives Systems, vol. 2, pp. 1640-1645, 2005. [Online]. Available: http://ieeexplore.ieee.org/lpdocs/epic03/wrapper.htm?arnumber=1619951

[3] B. J. A. M. Van Leersum, R. B. Timens, F. J. K. Buesink, and F. B. J. Leferink, "Time domain methods for the analysis of conducted interference on the power supply network of complex installations," IEEE International Symposium on Electromagnetic Compatibility, pp. 605-610, 2014.

[4] M. Pous, M. Azpúrua, and F. Silva, "Benefits of Full Time-Domain EMI Measurements for Large Fixed Installation," pp. 514-519, 2016.

[5] M. A. Azpúrua, M. Pous, and F. Silva, "Decomposition of Electromagnetic Interferences in the Time-Domain," IEEE Transactions on Electromagnetic Compatibility, vol. 58, no. 2, pp. 385-392, 2016.

[6] I. Setiawan, C. Keyer, M. Azpurua, F. Silva, and F. Leferink, "Timedomain Measurement Technique to Analyze Cyclic Short-Time Interference in Power Supply Networks," pp. 279-282, 2016.

[7] E. Puri and M. Monti, "Hidden Aspects in CISPR 16-1-1 Full Compliant Fast Fourier Transform EMI Receivers," pp. 34-39, 2016.

[8] _ _ "The Importance of Overload Revealing in EMI Receivers," 2017.

[9] C. Keyer, F. Buesink, and F. Leferink, "Mains Power Synchronous Conducted Noise Measurement in the 2 to $150 \mathrm{kHz}$ band," pp. 865869,2016

[10] N. Moonen, M. Gagic, F. Buesink, J. A. Ferreira, and F. Leferink, "Harmonic Cancellation in a Novel Multilevel Converter Topology for the Future Smart Grid," in Electromagnetic Compatibility (EMC), Signal and Power Integrity (SIPI), 2017 IEEE International Symposium on, Washington, 2017, p. to be published.

[11] N. Moonen, F. Buesink, and F. Leferink, "EMI Reduction in SPWM Driven SiC Converter Based on Carrier Frequency Selection," in 2017 International Symposium on Electromagnetic Compatibility - EMC EUROPE, Angers, 2017, p. to be published. 